\title{
Splitting obstructions and properties of objects in the Nil categories
}

\author{
by
}

\author{
Tadeusz Koźn i e ws ki (Warszawa)
}

\begin{abstract}
We show that the objects of Bass-Farrell categories which represent 0 in the corresponding Nil groups are precisely those which are stably triangular. This extends to Waldhausen's Nil group of the amalgamated free product with index 2 factors. Applications include a description of Cappell's special UNil group and reformulations of those splitting and fibering theorems which use the Nil groups.
\end{abstract}

1. Introduction. We start with a short review of the algebraic sources of different types of Nil groups. The following theorems relate them to the $K$-groups and $L$-groups.

Theorem 1.1 (Bass [Bs], Farrell, Hsiang [F], [FH1], [FH2]). Let $\alpha: R \rightarrow$ $R$ be a ring automorphism and let $R_{\alpha}\left[u, u^{-1}\right]$ be the $\alpha$-twisted ring of Laurent polynomials. Then there exists a subgroup $\widetilde{\operatorname{Nil}}(R, \alpha) \subset K_{1}\left(R_{\alpha}\left[u, u^{-1}\right]\right)$ with the property that $\widetilde{\operatorname{Nil}}(R, \alpha) \oplus \widetilde{\operatorname{Nil}}\left(R, \alpha^{-1}\right)$ is a direct summand and there is an exact sequence

$$
K_{1}(R) \stackrel{1-\alpha_{*}}{\longrightarrow} K_{1}(R) \rightarrow \frac{K_{1}\left(R_{\alpha}\left[u, u^{-1}\right]\right)}{\widetilde{\operatorname{Nil}}(R, \alpha) \oplus \widetilde{\operatorname{Nil}}\left(R, \alpha^{-1}\right)} \rightarrow K_{0}(R) \stackrel{1-\alpha_{*}}{\longrightarrow} K_{0}(R) .
$$

In particular if $a: H \rightarrow H$ is a group automorphism and $G=H \times_{a} \mathbb{Z}$, then for $R=\mathbb{Z} H$, a induces an automorphism $\alpha: R \rightarrow R, \mathbb{Z} G \cong R_{\alpha}\left[u, u^{-1}\right]$ and we have an exact sequence

$$
\mathrm{Wh}(H) \stackrel{1-\alpha_{*}}{\longrightarrow} \mathrm{Wh}(H) \rightarrow \frac{\mathrm{Wh}(G)}{\widetilde{\operatorname{Nil}}(R, \alpha) \oplus \widetilde{\operatorname{Nil}}\left(R, \alpha^{-1}\right)} \rightarrow \widetilde{K}_{0}(R) \stackrel{1-\alpha_{*}}{\longrightarrow} \widetilde{K}_{0}(R) .
$$

Also, for $\alpha=1$ we have $\widetilde{\operatorname{Nil}}(R, \alpha) \cong N K_{1}(R)$ of Bass.

1991 Mathematics Subject Classification: 19D35, 57R67, 57R80.

Research partially supported by KBN grant 2 P03A 01113. 
Theorem 1.2 (Waldhausen [W1], [W2]). For any two R-bimodules $B_{1}$, $B_{2}$ which are free both as left and as right $R$-modules there exists an abelian group $\widetilde{\operatorname{Nil}}\left(R ; B_{1}, B_{2}\right)$ with the following property. If $H$ is a subgroup of $G_{1}, G_{2}$ and $G=G_{1} *_{H} G_{2}, R=\mathbb{Z} H, B_{i}=\mathbb{Z}\left(G_{i} \backslash H\right)$ for $i=1,2$, then $\widetilde{\operatorname{Nil}}\left(R ; B_{1}, B_{2}\right)$ is a direct summand of $\mathrm{Wh}(G)$ and there is an exact sequence

$$
\begin{aligned}
\mathrm{Wh}(H) \rightarrow \mathrm{Wh}\left(G_{1}\right) \oplus \mathrm{Wh}\left(G_{2}\right) & \rightarrow \frac{\mathrm{Wh}(G)}{\widetilde{\operatorname{Nil}}\left(R ; B_{1}, B_{2}\right)} \\
& \rightarrow \widetilde{K}_{0}(\mathbb{Z} H) \rightarrow \widetilde{K}_{0}\left(\mathbb{Z} G_{1}\right) \oplus \widetilde{K}_{0}\left(\mathbb{Z} G_{2}\right) .
\end{aligned}
$$

Theorem 1.3 (Cappell [C1], [C2]). For any two R-bimodules $B_{1}, B_{2}$ with involution which are free both as left and right $R$-modules and for each natural number $n$ there exists an abelian group $\operatorname{UNil}_{n}^{h}\left(R ; B_{1}, B_{2}\right)$ such that for $G=G_{1} *_{H} G_{2}, R=\mathbb{Z} H$ and $B_{i}=\mathbb{Z}\left(G_{i} \backslash H\right)$ for $i=1,2$ there exists $a$ split monomorphism $\varrho: \operatorname{UNil}_{n}^{h}\left(R ; B_{1}, B_{2}\right) \rightarrow L_{n}^{h}(G)$ with the property that if $H^{i}\left(\mathbb{Z} / 2 ; \operatorname{ker}\left(\widetilde{K}_{0}(\mathbb{Z} H) \rightarrow \widetilde{K}_{0}\left(\mathbb{Z} G_{1}\right) \oplus \widetilde{K}_{0}\left(\mathbb{Z} G_{2}\right)\right)\right)=0$ for all $i$ then there exists an exact sequence

$\ldots \rightarrow L_{n}^{h}(H) \rightarrow L_{n}^{h}\left(G_{1}\right) \oplus L_{n}^{h}\left(G_{2}\right) \rightarrow \frac{L_{n}^{h}(G)}{\mathrm{UNil}_{n}^{h}\left(R ; B_{1}, B_{2}\right)} \rightarrow L_{n-1}^{h}(H) \rightarrow \ldots$

Cappell also defines a group $\operatorname{UNil}_{n}^{s}\left(R ; B_{1}, B_{2}\right)$ which is similarly related to $L_{n}^{s}(G)$.

One of the geometric reasons for the introduction of the Nil groups were various kinds of splitting problems. Let $Y$ be a closed $n$-dimensional manifold with a codimension 1 submanifold $X$. Let $f: M \rightarrow Y$ be a homotopy equivalence from a manifold $M$ to $Y$. Then $f$ is called splittable along $X$ if it is homotopic to a map $g: M \rightarrow Y$ which is transverse regular to $X$, so that $N=g^{-1}(X)$ is a manifold and $g$ restricted to $N$ is also a homotopy equivalence. If $f$ is h-cobordant to such a $g$ we say that $f$ is $h$-splittable. The following theorems give necessary and sufficient conditions for a map to be (h)-splittable.

Theorem 1.4 ([FH2]). Let $n \geq 6$ and let $\pi_{1}(Y)=H \times_{a} \mathbb{Z}$ with $\pi_{1}(X)$ $=H$. Then $f: M \rightarrow Y$ is splittable along $X \Leftrightarrow p(\tau(f))=0 \in \widetilde{\operatorname{Nil}}(R, \alpha)$, where $\tau(f) \in \mathrm{Wh}\left(H \times{ }_{a} \mathbb{Z}\right)$ is the torsion of $f, R=\mathbb{Z} H, \alpha: R \rightarrow R$ is induced by $a$ and $p: \operatorname{Wh}\left(H \times_{a} \mathbb{Z}\right) \rightarrow \widetilde{\operatorname{Nil}}(R, \alpha)$ is the projection map.

TheOREM $1.5([\mathrm{C} 2])$. Let $n \geq 6$ and let $\pi_{1}(Y)=G_{1} *_{H} G_{2}$ with $\pi_{1}(X)=$ $H$. Then for every homotopy equivalence $f: M \rightarrow Y$ there exist obstructions $\chi^{h}(f) \in \operatorname{UNil}_{n}^{h}\left(R ; B_{1}, B_{2}\right)$ and $\chi^{s}(f) \in \operatorname{UNil}_{n}^{s}\left(R ; B_{1}, B_{2}\right)$ with the properties:

(a) $f$ is h-splittable along $X \Leftrightarrow[\partial(\tau(f))]=0 \in H^{n}\left(\mathbb{Z} / 2 ; \operatorname{ker}\left(\widetilde{K}_{0}(\mathbb{Z} H) \rightarrow\right.\right.$ $\left.\left.\widetilde{K}_{0}\left(\mathbb{Z} G_{1}\right) \oplus \widetilde{K}_{0}\left(\mathbb{Z} G_{2}\right)\right)\right)$ and $\chi^{h}(f)=0$. 
(b) $f$ is splittable along $X \Leftrightarrow \tau(f) \in \operatorname{im}\left(\mathrm{Wh}\left(G_{1}\right) \oplus \operatorname{Wh}\left(G_{2}\right)\right)$ and $\chi^{s}(f)$ $=0$.

Here, as before, $\tau(f)$ is the torsion of $f, R=\mathbb{Z} H, B_{i}=\mathbb{Z}\left(G_{i} \backslash H\right)$ and $\partial$ is the boundary map of 1.2 .

The UNil groups are closely related to the structure sets of a manifold. Cappell shows that if $\pi_{1}(Y)=G=G_{1} *_{H} G_{2}$, then for $x=h, s$ the group $\mathrm{UNil}_{n}^{x}\left(R ; B_{1}, B_{2}\right)$ acts freely on the structure set $\mathcal{S}^{x}(Y)$. In particular if $Y$ is aspherical with universal cover $\mathbb{R}^{n}$ then free actions of $G$ on $\mathbb{R}^{n}$ are h-rigid (resp. rigid) only if $\mathrm{UNil}_{n}^{h}\left(R ; B_{1}, B_{2}\right)=0\left(\mathrm{UNil}_{n}^{s}\left(R ; B_{1}, B_{2}\right)=0\right)$. For some of the newest results on the Nil groups and their applications see [Bd], [CK], $[\mathrm{KS}],[\mathrm{R}]$.

2. Triangular objects in the Farrell category. In this section we analyze the properties of the Farrell category $\operatorname{Nil}(R, \alpha)$.

Let $R$ be a ring and let $\alpha: R \rightarrow R$ be an automorphism of $R$. We say that a map $\varphi: P \rightarrow Q$ of right $R$-modules is $\alpha$-linear if $\varphi$ is additive and $\varphi(z r)=\varphi(z) \alpha(r)$ for each $z \in P, r \in R$. An object of the category $\operatorname{Nil}(R, \alpha)$ is a pair $(P, \nu)$, where $P$ is a stably free, finitely generated right $R$-module and $\nu: P \rightarrow P$ is an $\alpha$-linear endomorphism which is nilpotent, i.e. $\nu^{n}=0$ for some $n$. Let $R_{\alpha}[u]$ denote the $\alpha$-twisted polynomial ring, with $r u=u \alpha(r)$ and let $R_{n}=R_{\alpha}[u] /\left(u^{n}\right)$. Then the objects of $\operatorname{Nil}(R, \alpha)$ in which $\nu^{n}=0$ are precisely those right $R_{n}$-modules which are stably free as $R$-modules.

A morphism in $\operatorname{Nil}(R, \alpha)$ from $(P, \nu)$ to $\left(P^{\prime}, \nu^{\prime}\right)$ is an $R$-homomorphism $f: P \rightarrow P^{\prime}$ such that $\nu^{\prime} f=f \nu$. A sequence of morphisms in $\operatorname{Nil}(R, \alpha)$ is exact if it is exact in the category of $R$-modules, after we forget about the endomorphisms.

Let $\operatorname{Nil}(R, \alpha):=K_{0}(\operatorname{Nil}(R, \alpha))$. This means that $\operatorname{Nil}(R, \alpha)$ is the Grothendieck group of the isomorphism classes of objects of $\operatorname{Nil}(R, \alpha)$ modulo the relation generated by short exact sequences. Let $\mathbf{F}_{R}$ be the category of finitely generated, free right $R$-modules. The functor $\iota: \mathbf{F}_{R} \rightarrow \operatorname{Nil}(R, \alpha)$ which assigns to a module $P$ the object $(P, 0)$ induces on $K_{0}$ a group homomorphism $\iota_{*}: \mathbb{Z} \rightarrow \operatorname{Nil}(R, \alpha)$. One then defines $\widetilde{\operatorname{Nil}}(R, \alpha):=\operatorname{coker} \iota_{*}$.

We say that an object $x=(P, \nu)$ in $\operatorname{Nil}(R, \alpha)$ is triangular if there is a filtration $0=E_{0} \subset E_{1} \subset \ldots \subset E_{n}=P$ such that for each $i$ the module $E_{i+1} / E_{i}$ is free and $\nu\left(E_{i}\right) \subset E_{i-1}$. For example, $R_{n}$ with the endomorphism given by the right multiplication by $u$ is triangular. Also, if $P$ is a free right $R$-module, then $P \otimes_{R} R_{n}$ with $\nu\left(\sum p_{i} u^{i}\right)=\sum p_{i} u^{i+1}$ is triangular.

Lemma 2.1 (Farrell $[\mathrm{F}]$ ). If $x$ is triangular, then $[x]=0 \in \widetilde{\operatorname{Nil}}(R, \alpha)$.

We call $x$ a stably triangular object if there exists a triangular object $t$ such that $x \oplus t$ is triangular. 
Corollary 2.2. If $x$ is stably triangular, then $[x]=0$.

Lemma 2.3. If $0 \rightarrow t^{\prime} \rightarrow t \rightarrow t^{\prime \prime} \rightarrow 0$ is an exact sequence of objects of $\operatorname{Nil}(R, \alpha)$ and $t^{\prime}, t^{\prime \prime}$ are triangular, then $t$ is triangular.

Proof. A triangular filtration of $t^{\prime \prime}$ pulled back to $t$ and combined with a triangular filtration of $t^{\prime}$ provides a triangular filtration of $t$.

Following Waldhausen [W1], we say that $y$ is a suspension of $x$ if there is an exact sequence $0 \rightarrow y \rightarrow t \rightarrow x \rightarrow 0$ of objects in $\operatorname{Nil}(R, \alpha)$ with $t$ triangular. If $t$ is $R_{n}$-free, then we say that $y$ is an $R_{n}$-suspension of $x$.

LEMMA 2.4. (i) Each object has a suspension.

(ii) Triangular objects admit $R_{n}$-suspensions which are triangular.

Pr o of. (i) Let $x=(P, \nu)$. We can assume that $P$ is free, if not we can add to $x$ a free module with a trivial endomorphism and consider the natural projection from the resulting sum to $x$. Now $P \otimes_{R} R_{n}$ is free and maps epimorphically onto $x$ by $\varepsilon\left(\sum p_{i} u^{i}\right)=\sum \nu^{i}\left(p_{i}\right)$. So ker $\varepsilon$ is a suspension of $x$.

(ii) Let $t$ be a triangular object. We proceed by induction on $l(t)$, the length of the shortest triangular filtration of $t$. If $l(t)=1$, then $\operatorname{ker}(\varepsilon$ : $\left.P \otimes_{R} R_{n} \rightarrow P\right)=P u+\ldots+P u^{n-1}$ is a stably free $R_{n-1}$-module, and therefore triangular.

If $l(t)=k$, then there is an exact sequence $0 \rightarrow t^{\prime} \rightarrow t \rightarrow t^{\prime \prime} \rightarrow 0$ where $l\left(t^{\prime \prime}\right)=1$ and $l\left(t^{\prime}\right)=k-1$. Because $\otimes_{R} R_{n}$ is an exact functor we can use the construction of (i) to get an exact sequence $0 \rightarrow u^{\prime} \rightarrow u \rightarrow u^{\prime \prime} \rightarrow 0$ of $R_{n}$-suspensions. By the induction hypothesis $u^{\prime}$ and $u^{\prime \prime}$ are now triangular, so $u$ is also triangular, by Lemma 2.3 .

Lemma 2.5. A suspension of a triangular object is stably triangular.

Proof. Let $y$ be a suspension of $x$, i.e. $y=\operatorname{ker} \varepsilon_{1}$ where $0 \rightarrow \operatorname{ker} \varepsilon_{1} \rightarrow$ $t_{1} \rightarrow x \rightarrow 0$ is exact and $t_{1}$ is triangular. We have to show that $y$ is stably triangular.

Let $z$ be a triangular $R_{n}$-suspension of $x$, where $n \geq l(x), l(t)$. So $z=$ ker $\varepsilon_{2}$ for an exact sequence $0 \rightarrow \operatorname{ker} \varepsilon_{2} \rightarrow t_{2} \rightarrow x \rightarrow 0$ with $t_{2} R_{n}$-free. Such a $z$ exists by Lemma 2.4(ii).

We define $t_{1} \times{ }_{x} t_{2}$ in $\operatorname{Nil}(R, \alpha)$ as $(P, \mu)$ where

$$
P=\left\{\left(c_{1}, c_{2}\right) \in T_{1} \times T_{2} \mid \varepsilon_{1}\left(c_{1}\right)=\varepsilon_{2}\left(c_{2}\right)\right\}, \quad \mu=\nu_{1} \times\left.\nu_{2}\right|_{P} .
$$

This gives a commutative diagram with exact columns and rows: 


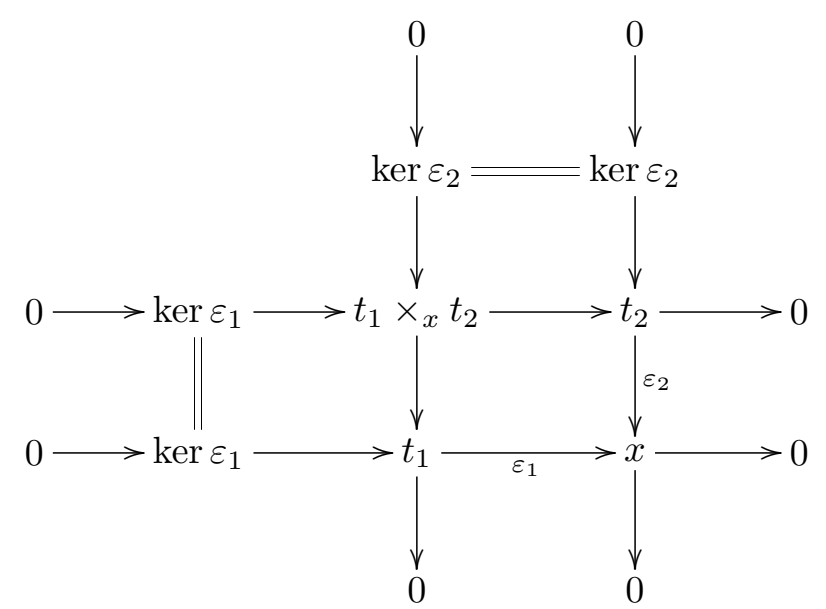

Since $t_{1}$ and $\operatorname{ker} \varepsilon_{2}$ are triangular, Lemma 2.3 implies that $t_{1} \times_{x} t_{2}$ is triangular. Moreover $t_{2}$ is $R_{n}$-free, so the row containing $t_{1} \times_{x} t_{2}$ splits, and $t_{1} \times_{x} t_{2}=\operatorname{ker} \varepsilon_{1} \oplus t_{2}$. We conclude that ker $\varepsilon_{1}$ is stably triangular.

LEMMA 2.6. If an object of $\mathbf{N i l}(R, \alpha)$ has a triangular suspension, then it is stably triangular.

Proof. For any object $x=(P, \nu)$ in $\operatorname{Nil}(R, \alpha)$ let $P^{*}=\operatorname{Hom}_{R}(P, R)$ and let $\nu^{*}: P^{*} \rightarrow P^{*}$ be given by $\nu^{*}(\varphi)=\alpha^{-1} \circ \varphi \circ \nu$. Then $P^{*}$ has the structure of a left $R$-module $((r \varphi)(c)=r \varphi(c))$ and $\nu^{*}$ is an $\alpha^{-1}$-linear endomorphism. This gives a (contravariant) functor $\#: \mathbf{N i l}(R, \alpha) \rightarrow \mathbf{N i l}^{\prime}\left(R, \alpha^{-1}\right)$ where $(P, \nu)^{\#}=\left(P^{*}, \nu^{*}\right)$ and where $\mathbf{N i l}^{\prime}(R, \beta)$ denotes the analog of $\mathbf{N i l}(R, \beta)$ with left modules used instead of right modules. Then $x \in \operatorname{Nil}(R, \alpha)$ is triangular $\Leftrightarrow x^{\#}$ is triangular.

Now let $x$ be an object in $\operatorname{Nil}(R, \alpha)$ with a triangular suspension. This means that we have an exact sequence

$$
0 \rightarrow t_{1} \rightarrow t \rightarrow x \rightarrow 0
$$

where $t$ and $t_{1}$ are triangular. Applying \# we get a sequence

$$
0 \rightarrow x^{\#} \rightarrow t^{\#} \rightarrow t_{1}^{\#} \rightarrow 0
$$

which is also exact (because our sequences split as sequences of $R$-modules). From Lemma 2.5 it follows that $x^{\#}$ is stably triangular, so $x$ itself is stably triangular.

As a corollary we get the following

Proposition 2.7. If in an exact sequence of objects in $\operatorname{Nil}(R, \alpha)$

$$
0 \rightarrow x \rightarrow y \rightarrow z \rightarrow 0
$$

two objects are stably triangular, then the third is also stably triangular.

THEOREM 2.8. If $x_{1}$ is a suspension of $x_{2}$ then $x_{1} \oplus x_{2}$ is stably triangular. 
Pr o o f. Consider exact sequences

$$
0 \rightarrow x_{1} \rightarrow x \rightarrow x_{2} \rightarrow 0 \quad \text { and } \quad 0 \rightarrow y_{i} \rightarrow t_{i} \rightarrow x_{i} \rightarrow 0
$$

for $i=1,2$, with $x, t_{1}, t_{2}$ triangular. To show that $x_{1} \oplus x_{2}$ is stably triangular we use the following construction which is due to Waldhausen [W1], [W2]. There exists a commutative diagram of objects in $\operatorname{Nil}(R, \alpha)$ with exact rows:

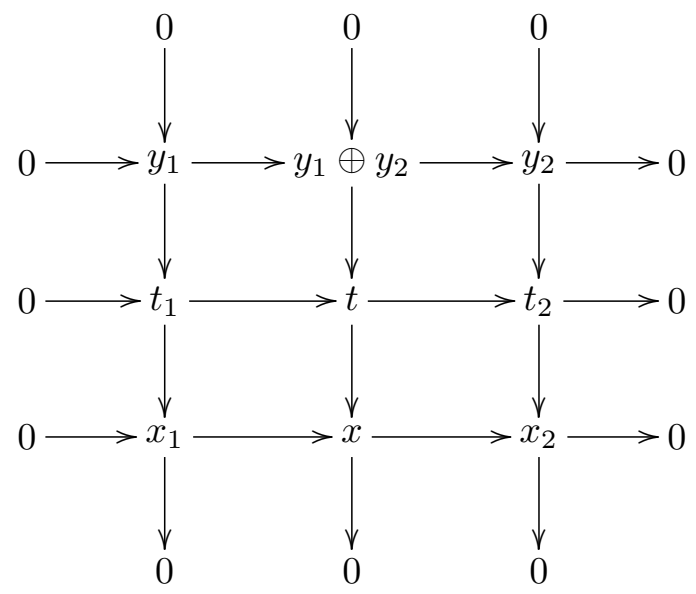

where for $x=\left(P_{x}, \nu_{x}\right), x_{i}=\left(P_{x_{i}}, \nu_{x_{i}}\right), t_{i}=\left(P_{t_{i}}, \nu_{t_{i}}\right), i=1,2$, one defines $t=\left(P_{t}, \nu_{t}\right)$ by

$$
P_{t}=P_{t_{1}} \oplus P_{t_{2}}, \quad \nu_{t}=\left(\begin{array}{cc}
\nu_{t_{1}} & c \\
0 & \nu_{t_{2}}
\end{array}\right)
$$

with $c: P_{t_{2}} \rightarrow P_{t_{1}}$ specified as follows. We have $P_{x} \cong P_{x_{1}} \oplus P_{x_{2}}$ and $\nu_{x}=\left(\begin{array}{cc}\nu_{x_{1}} & \gamma \\ 0 & \nu_{x_{2}}\end{array}\right)$ where $\gamma: P_{x_{2}} \rightarrow P_{x_{1}}$. Then $P_{t_{i}}=P_{y_{i}} \oplus P_{x_{i}}$ for $i=1,2$ and $c:=\left(\begin{array}{ll}0 & 0 \\ 0 & \gamma\end{array}\right)$.

All the rows and columns are exact. Also, since $t_{1}, t_{2}$ are triangular, $t$ is triangular (Lemma 2.3). Now $x, t$ triangular imply that $y_{1} \oplus y_{2}$ is stably triangular (Lemma 2.5). Finally $y_{1} \oplus y_{2}$ and $t_{1} \oplus t_{2}$ stably triangular make $x_{1} \oplus x_{2}$ stably triangular (Lemma 2.6).

We now give another description of $\widetilde{\operatorname{Nil}}(R, \alpha)$. Let $N$ be the set of isomorphism classes of objects in $\operatorname{Nil}(R, \alpha)$. Then $N$ has the structure of an abelian semigroup with addition given by $\oplus$. We introduce an equivalence relation in $N$ by

$$
x_{1} \sim x_{2} \Leftrightarrow \exists t_{1}, t_{2} \text { triangular such that } x_{1} \oplus t_{1}=x_{2} \oplus t_{2} .
$$

REMARK 2.9. (a) $x \sim 0 \Leftrightarrow x$ is stably triangular. of $x$.

(b) $N / \sim$ is an abelian group. The inverse of $x$ is given by a suspension

(c) If $0 \rightarrow x_{1} \rightarrow x \rightarrow x_{2} \rightarrow 0$ is exact, then $\left[x_{1}\right]+\left[x_{2}\right]=[x]$ in $N / \sim$. 
Proof. (a) is clear. To see (b) note that if $y$ is a suspension of $x$, then (by Theorem 2.8) $x \oplus y$ is stably triangular, so $[x]+[y]=0$ in $N / \sim$.

(c) Let $y_{i}$ be a suspension of $x_{i}, i=1,2$. By the construction given in Theorem 2.8 it follows that $y_{1} \oplus y_{2}$ is a suspension of $x$. So $[x]=-\left[y_{1} \oplus y_{2}\right]=$ $-\left(-\left[x_{1}\right]-\left[x_{2}\right]\right)=\left[x_{1}\right]+\left[x_{2}\right]$.

Corollary 2.10. The natural epimorphism $j: N / \sim \rightarrow \widetilde{\operatorname{Nil}}(R, \alpha)$ is an isomorphism.

As a consequence we get

TheOREM 2.11. Two objects $x_{1}, x_{2}$ of the category $\mathbf{N i l}(R, \alpha)$ represent the same element of $\widetilde{\mathrm{Nil}}(R, \alpha) \Leftrightarrow$ there exist triangular objects $t_{1}, t_{2}$ such that $x_{1} \oplus t_{1} \cong x_{2} \oplus t_{2}$.

Corollary 2.12. An object $x$ of $\operatorname{Nil}(R, \alpha)$ represents $0 \in \widetilde{\operatorname{Nil}}(R, \alpha) \Leftrightarrow$ $x$ is stably triangular.

3. Waldhausen's Nil group. In this section we show how to extend the results of $\S 2$ to some other types of Nil categories.

For any two $R$-bimodules $B_{1}, B_{2}$ which are free both as left and as right $R$-modules let $\operatorname{Nil}\left(R ; B_{1}, B_{2}\right)$ be the following category. An object of $\operatorname{Nil}\left(R ; B_{1}, B_{2}\right)$ is a quadruple $(P, Q, p, q)$ where $P, Q$ are finitely generated, stably free right $R$-modules and $p: P \rightarrow Q \otimes_{R} B_{1}$ and $q: Q \rightarrow P \otimes_{R} B_{2}$ are $R$-homomorphisms which satisfy the following nilpotency condition. There exist filtrations $0=P_{0} \subset P_{1} \subset \ldots \subset P_{n}=P$ and $0=Q_{0} \subset Q_{1} \subset \ldots \subset$ $Q_{n}=Q$ so that $p\left(P_{i}\right) \subset Q_{i-1} \otimes_{R} B_{1}$ and $q\left(Q_{i}\right) \subset P_{i-1} \otimes_{R} B_{2}$ for all $i \geq 1$.

A morphism $(P, Q, p, q) \rightarrow\left(P^{\prime}, Q^{\prime}, p^{\prime}, q^{\prime}\right)$ is a pair of $R$-homomorphisms $f: P \rightarrow P^{\prime}, g: Q \rightarrow Q^{\prime}$ so that one has the commutative diagrams
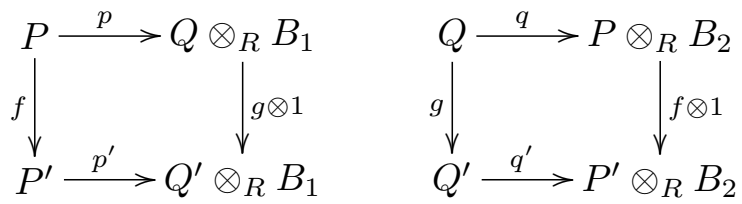

Forming $K_{0}$ of this category we get the natural homomorphism

$$
\mathbb{Z} \oplus \mathbb{Z} \stackrel{j}{\longrightarrow} K_{0}\left(\mathbf{N i l}\left(R ; B_{1}, B_{2}\right)\right)
$$

defined by $j(k, l)=\left(R^{k}, R^{l}, 0,0\right)$ for $k, l \geq 0$. Waldhausen defines $\widetilde{\operatorname{Nil}}\left(R ; B_{1}, B_{2}\right)$ as the cokernel of $j$. His notation for this group in [W1] is $\widetilde{C}\left(R ; B_{1}, B_{2}\right)$.

We will discuss the group $\widetilde{\operatorname{Nil}}\left(R ; B_{1}, B_{2}\right)$ in the special case when there are ring automorphisms $\beta_{1}, \beta_{2}: R \rightarrow R$ such that: 
(a) $B_{1}, B_{2}$ are isomorphic to $R$ as left modules via isomorphisms

$$
B_{1} \stackrel{\Phi_{1}}{\longrightarrow} R \stackrel{\Phi_{2}}{\longleftarrow} B_{2},
$$

(b) the map $\Phi_{i}$ is $\beta_{i}$-linear, i.e. $\Phi_{i}(z r)=\Phi_{i}(z) \beta_{i}(r)$ for all $z \in B_{i}, r \in R$ and $i=1,2$.

When $B_{1}, B_{2}$ satisfy conditions (a), (b) we write $R_{\beta_{i}}$ for $B_{i}$ and $\widetilde{\operatorname{Nil}}\left(R, \beta_{1}, \beta_{2}\right)$ for $\widetilde{\operatorname{Nil}}\left(R ; B_{1}, B_{2}\right)$.

The importance of this special case follows from the following remark. Let

$$
1 \rightarrow C \rightarrow \Gamma \stackrel{q}{\longrightarrow} D_{\infty} \rightarrow 1
$$

be an exact sequence of groups, where $D_{\infty}=\left\langle s_{1}, s_{2}: s_{1}^{2}=s_{2}^{2}=1\right\rangle$ is the infinite dihedral group. Set $R=\mathbb{Z} C, \Gamma_{i}=q^{-1}\left(\left\langle s_{i}\right\rangle\right)$ and $B_{i}=\mathbb{Z}\left(\Gamma_{i} \backslash C\right)$ for $i=1,2$. Then $\Gamma=\Gamma_{1} *_{C} \Gamma_{2}$ and $B_{i}$ is isomorphic to $R$ as a left $R$ module by the isomorphism $\Phi_{i}: B_{i} \rightarrow R, \Phi_{i}(x)=x b_{i}$ for $x \in B_{i}$, where $b_{i}$ is some chosen element of $\Gamma_{i} \backslash C$ for $i=1,2$. The elements $b_{1}, b_{2}$ specify two automorphisms $\beta_{i}$ of $R: \beta_{i}(r)=b_{i}^{-1} r b_{i}$ for $r \in R$. As a map of right $R$-modules, $\Phi_{i}$ is $\beta_{i}$-linear. Therefore in this case we get $\widetilde{\operatorname{Nil}}\left(R ; B_{1}, B_{2}\right)=$ $\widetilde{\operatorname{Nil}}\left(R, \beta_{1}, \beta_{2}\right)$.

We say that an object $(P, Q, p, q)$ of $\operatorname{Nil}\left(R ; B_{1}, B_{2}\right)$ is triangular if there exist filtrations $0=P_{0} \subset P_{1} \subset \ldots \subset P_{n}=P$ and $0=Q_{0} \subset Q_{1} \subset \ldots \subset$ $Q_{n}=Q$ so that $p\left(P_{i}\right) \subset Q_{i-1} \otimes_{R} B_{1}$ and $q\left(Q_{i}\right) \subset P_{i-1} \otimes_{R} B_{2}$ for all $i \geq 1$ with the extra property that for all $i$ the modules $P_{i} / P_{i-1}$ and $Q_{i} / Q_{i-1}$ are free. An object $x$ is called stably triangular if $x \oplus t$ is triangular for some triangular $t$. Our goal is to show that an object in $\operatorname{Nil}\left(R ; R_{\beta_{1}}, R_{\beta_{2}}\right)$ represents 0 in $\widetilde{\operatorname{Nil}}\left(R, \beta_{1}, \beta_{2}\right)$ precisely when it is stably triangular. In order to do this we first observe that $\operatorname{Nil}\left(R ; R_{\beta_{1}}, R_{\beta_{2}}\right)$ is isomorphic to another category, $\operatorname{Nil}\left(R, \beta_{1}, \beta_{2}\right)$, which we now describe.

An object of $\operatorname{Nil}\left(R, \beta_{1}, \beta_{2}\right)$ is a quadruple $(P, Q, p, q)$, where $P, Q$ are finitely generated, stably free right $R$-modules, $p: P \rightarrow Q$ is $\beta_{1}$-linear, $q: Q \rightarrow P$ is $\beta_{2}$-linear and $q p: P \rightarrow P$ is nilpotent $\left((q p)^{n}=0\right.$ for some $n)$. A morphism from $(P, Q, p, q)$ to $\left(P^{\prime}, Q^{\prime}, p^{\prime}, q^{\prime}\right)$ is a pair $(f, g)$, where $f: P \rightarrow P^{\prime}$ and $g: Q \rightarrow Q^{\prime}$ are $R$-homomorphisms and $p^{\prime} f=g p, q^{\prime} g=f q$. An object of $\operatorname{Nil}\left(R, \beta_{1}, \beta_{2}\right)$ is triangular if there exist filtrations $0=P_{0} \subset$ $P_{1} \subset \ldots \subset P_{n}=P$ and $0=Q_{0} \subset Q_{1} \subset \ldots \subset Q_{n}=Q$ so that for all $i$ $p\left(P_{i}\right) \subset Q_{i-1}, q\left(Q_{i}\right) \subset P_{i-1}$ and the modules $P_{i} / P_{i-1}$ and $Q_{i} / Q_{i-1}$ are free. An object is called stably triangular if it becomes triangular after adding some triangular object.

Note that for any right $R$-module $M$ there is a $\beta_{i}$-linear isomorphism $M \otimes_{R} R_{\beta_{i}} \rightarrow M$ given by $x \otimes s \mapsto x \Phi_{i}(s)$. So any $R$-homomorphism $P \rightarrow Q \otimes_{R} R_{\beta_{1}}$ specifies uniquely a $\beta_{1}$-linear homomorphism $P \rightarrow Q$. Sim- 
ilarly, any $R$-homomorphism $Q \rightarrow P \otimes_{R} R_{\beta_{2}}$ determines a $\beta_{2}$-linear homomorphism $Q \rightarrow P$. This provides an obvious isomorphism of the categories $\operatorname{Nil}\left(R ; R_{\beta_{1}}, R_{\beta_{2}}\right)$ and $\operatorname{Nil}\left(R, \beta_{1}, \beta_{2}\right)$ under which triangular objects go to triangular objects.

We now give a characterization of the objects representing 0 in $\widetilde{\operatorname{Nil}}\left(R, \beta_{1}, \beta_{2}\right)$, by showing that for any ring $R$ and any two automorphisms $\beta_{1}, \beta_{2}$ of $R$ the following holds.

THEOREM 3.1. Two objects $x, y$ in $\operatorname{Nil}\left(R, \beta_{1}, \beta_{2}\right)$ represent the same element in $\widetilde{\operatorname{Nil}}\left(R, \beta_{1}, \beta_{2}\right) \Leftrightarrow x \oplus t$ is isomorphic to $y \oplus t^{\prime}$ for some triangular objects $t, t^{\prime}$.

Proof. If $(P, Q, p, q)$ is an object of $\operatorname{Nil}\left(R, \beta_{1}, \beta_{2}\right)$ we can add a triangular object $t$ to it if necessary, so that $x \oplus t=\left(P^{\prime}, Q^{\prime}, p^{\prime}, q^{\prime}\right)$ and $P^{\prime} \cong Q^{\prime}$ as right $R$-modules ( $t$ has the form $\left(R^{k}, 0,0,0\right)$ or $\left(0, R^{k}, 0,0\right)$ ). Therefore it is sufficient to prove 3.1 in the full subcategory $\operatorname{Nil}^{\prime}\left(R, \beta_{1}, \beta_{2}\right)$ whose objects $(P, Q, p, q)$ satisfy $P \cong Q$.

Let $S$ be the ring $R \times R$. Let $\alpha: S \rightarrow S$ be the ring automorphism

$$
\alpha(x, y)=\left(\beta_{2}(y), \beta_{1}(x)\right) \quad \forall(x, y) \in R \times R .
$$

Let $e=(1,0)$ and $f=(0,1)$ in $S$. If $F$ is a free right $S$-module, say $F \cong S^{k}$, then $P=F e$ and $Q=F f$ are right free $R$-modules and $F=P \oplus Q$.

If $\nu: F \rightarrow F$ is an $\alpha$-linear map, then $\nu(P) \subset Q, \nu(Q) \subset P$ and $\nu$ can be written as $\left(\begin{array}{ll}0 & q \\ p & 0\end{array}\right): P \oplus Q \rightarrow P \oplus Q$ where $p: P \rightarrow Q$ is $\beta_{1}$-linear and $q: Q \rightarrow P$ is $\beta_{2}$-linear. Also, if $\nu^{2 n}=0$ then $(p q)^{n}=0$.

So an object $(F, \nu)$ in $\operatorname{Nil}(S, \alpha)$ determines an object $(P, Q, p, q)$ in $\operatorname{Nil}^{\prime}\left(R, \beta_{1}, \beta_{2}\right)$. Conversely, if $(P, Q, p, q)$ is an object in $\operatorname{Nil}^{\prime}\left(R, \beta_{1}, \beta_{2}\right)$ then $F=P \oplus Q$ is a stably free right $S$-module with nilpotent $\alpha$-linear endomorphism $\nu=\left(\begin{array}{ll}0 & q \\ p & 0\end{array}\right)$.

This gives an equivalence of categories $E: \operatorname{Nil}(S, \alpha) \rightarrow \mathbf{N i l}^{\prime}\left(R, \beta_{1}, \beta_{2}\right)$. It preserves sums and exact sequences, and $E(t)$ is triangular if $t$ is. Conversely, suppose $E(t)=(P, Q, p, q)$ is triangular. By refining the filtration if necessary, we can assume that $P_{i} / P_{i-1}$ and $Q_{i} / Q_{i-1}$ have rank 1 for all $i \geq 1$. This means that $F_{i}:=P_{i} \oplus Q_{i}$ is a free $R \times R$-module and so is $F_{i} / F_{i-1}$. Moreover, $\nu\left(F_{i}\right) \subset F_{i-1}$. So $t$ itself is triangular.

It follows from the above discussion that $E$ induces an isomorphism $\widetilde{\operatorname{Nil}}(S, \alpha) \cong \widetilde{\operatorname{Nil}}\left(R, \beta_{1}, \beta_{2}\right)$. Our theorem now follows from 2.11 .

Corollary 3.2. An object in $\operatorname{Nil}\left(R, \beta_{1}, \beta_{2}\right)$ represents 0 in $\widetilde{\operatorname{Nil}}\left(R, \beta_{1}, \beta_{2}\right)$ $\Leftrightarrow$ it is stably triangular.

Cappell defines simple UNil forms as those UNil forms for which the corresponding objects in $\widetilde{\operatorname{Nil}}\left(R ; B_{1}, B_{2}\right)$ are trivial. 
Corollary 3.3. If $G=G_{1} *_{H} G_{2}$ with $\left|G_{i}: H\right|=2, R=\mathbb{Z} H, B_{i}=$ $\mathbb{Z}\left(G_{i} \backslash H\right)$ for $i=1,2$, then the simple $(-1)^{k}$ UNil forms over $\left(B_{1}, B_{2}\right)$ are pairs $\left(x_{1}, x_{2}\right)$ where $x_{i}=\left(P_{i}, \lambda_{i}, \mu_{i}\right)$ is a $(-1)^{k}$ Hermitian form over $B_{i}$ (in the sense of Wall [W1], [W2]), $P_{2}=P_{1}^{*}$ and $\left(P_{1}, P_{2}\right.$, ad $\lambda_{1}$, ad $\left.\lambda_{2}\right)$ is a stably triangular object of $\operatorname{Nil}\left(R ; B_{1}, B_{2}\right)$.

4. Applications. We now give examples of some geometric applications of the above theorems. Here is the main idea of getting them.

In the topological theorems which make use of the Nil groups one usually tries to carry out a geometric construction in order to prove a general statement. It turns out that the completion of the construction is not always possible. What one gets is a geometrically defined module with a nilpotent endomorphism. The construction can be completed if and only if this object represents 0 in the appropriate Nil group. One shows that the choices which are made during the attempt of the construction do not alter the class of the module and its endomorphism in the Nil group. This gives a Nil obstruction.

From Corollary 2.12 it now follows that to decide whether the construction can always be completed it is enough to attempt the construction, making any choices, and check if the resulting module with endomorphism is stably triangular. Below we give an example of the above method. It is based on the construction in $[\mathrm{F}]$. Similar examples, related to the splitting theorems 1.4, 1.5, can be obtained using [FH2], [C2], [C3].

Let $M$ be a closed, $n$-dimensional manifold with $n \geq 6$ and let $f: M \rightarrow$ $S^{1}$ be a continuous map. Assume that $f_{\#}: \pi_{1}(M) \rightarrow \mathbb{Z}$ is onto and let $H=\operatorname{ker} f_{\#}$. So $\pi_{1}(M)=H \times{ }_{a} \mathbb{Z}$ and $a$ induces an automorphism $\alpha$ of $\mathbb{Z} H$. Let $X$ be the covering space of $M$ corresponding to $H$. Assume that $X$ has the homotopy type of a finite CW complex. The group $\pi_{1}(M) / H \cong \mathbb{Z}$ acts on $X$. A closed, framed, $(n-1)$-dimensional submanifold $(N, \mu)$ of $M$ is called a splitting of $f$ if it corresponds to $f$ under the Pontryagin-Thom construction. Then $W=M \backslash$ (a tubular neighborhood of $N$ ) is a cobordism on $N$. Lift $(N, \mu)$ to a framed submanifold $(\widehat{N}, \widehat{\mu})$ of $X$. Then $X \backslash \widehat{N}$ has two components, say $A$ and $B$, where $B$ denotes the component to which $\widehat{\mu}$ points. Let $T$ be the generator of $\pi_{1}(M) / H$ for which $A \subset T(A)$. Then $T^{-1}$ induces an $\alpha$-linear endomorphism $T_{*}^{-1}$ of $H_{i}(X, A ; \mathbb{Z} H)$. Farrell shows that one can always find $(N, \mu)$ so that $H_{i}(X, A ; \mathbb{Z} H)=0$ for $i \leq 2$ and $H_{i}(X, B ; \mathbb{Z} H)=0$ for $i \leq n-3$, in which case $H_{3}(X, A ; \mathbb{Z} H)$ is a finitely generated, projective $\mathbb{Z} H$-module and $T_{*}^{-1}$ is nilpotent. Pick any such $(N, \mu)$ and denote $\left(H_{3}(X, A ; \mathbb{Z} H), T_{*}^{-1}\right)$ as $(P, \nu)$.

Then Farrell's results can be stated in the following way:

THEOREM 4.1. (a) There exists a splitting for which $W$ is an h-cobordism $\Leftrightarrow P$ is stably free and $(P, \nu)$ is stably triangular. 
Also, if these conditions are satisfied then the class $\tau(f)$ in $\mathrm{Wh}(H) /$ $\left(1-\alpha_{*}\right) \mathrm{Wh}(H)$ of the torsion of such an $h$-cobordism $W$ does not depend on the choice of the splitting.

(b) There exists a splitting for which $W$ is a product cobordism (equivalently: $f$ is homotopic to a locally trivial bundle map) $\Leftrightarrow$ the conditions of (a) hold and $\tau(f)=0$.

\section{References}

[Bd] B. Badzioch, $K_{1}$ of twisted polynomial rings, K-Theory 16 (1999), 29-34.

[Bs] H. Bass, Algebraic K-Theory, Benjamin, New York, 1968.

[C1] S. Cappell, Unitary nilpotent groups and Hermitian K-theory, Bull. Amer. Math. Soc. 80 (1974), 1117-1122.

[C2] - Manifolds with fundamental group a generalized free product, ibid. 80 (1974), 1193-1198.

[C3] —, A splitting theorem for manifolds, Invent. Math. 33 (1976), 69-170.

[CK] F. Connolly and T. Koźniewski, Nil groups in K-theory and surgery theory, Forum Math. 7 (1995), 45-76.

[F] F. T. Farrell, The obstruction to fibering a manifold over a circle, Indiana Univ. Math. J. 21 (1971), 315-346.

[FH1] F. T. Farrell and W. C. Hsiang, A formula for $K_{1} R_{\alpha}[T]$, in: Proc. Sympos. Pure Math. 17, Amer. Math. Soc., 1970, 192-218.

[FH2] - - - Manifolds with $\pi_{1}=G \times{ }_{\alpha} T$, Amer. J. Math. 95 (1973), 813-848.

[KS] S. Kwasik and R. Schultz, Unitary nilpotent groups and the stability of pseudoisotopies, Duke Math. J. 71 (1993), 871-887.

[R] A. Ranicki, Lower K- and L-Theory, Cambridge Univ. Press, 1992.

[W1] F. Waldhausen, Whitehead groups of generalized free products, preprint, 1969.

[W2] - Algebraic K-theory of generalized free products, Ann. of Math. 108 (1978), $135-256$.

Institute of Mathematics

Warsaw University

Banacha 2

02-097 Warszawa, Poland

E-mail: t.kozniewski@mimuw.edu.pl

Received 30 December 1997;

in revised form 9 February 1999 